\title{
Antigenicity and adhesiveness of a Plasmodium vivax VIR-E protein from Brazilian isolates
}

\author{
Ana Paula Schappo', Najara C Bittencourt², Leticia P Bertolla1', Sofia Forcellini', \\ Ana Beatriz lung Enembreck da Silva', Hellen Geremias dos Santos' ${ }^{1}$ João Henrique Gervásio', \\ Marcus VG Lacerda ${ }^{3,4}$, Stefanie CP Lopes ${ }^{3}$, Fabio TM Costa ${ }^{2}$, Letusa Albrecht ${ }^{1,2}{ }^{+}$ \\ ${ }^{1}$ Fundação Oswaldo Cruz-Fiocruz, Instituto Carlos Chagas, Laboratório de Pesquisa em Apicomplexa, Curitiba, PR, Brasil \\ ${ }^{2}$ Universidade Estadual de Campinas, Instituto de Biologia, Departamento de Genética, Evolução, Microbiologia e Imunologia, \\ Campinas, SP, Brasil \\ ${ }^{3}$ Fundação Oswaldo Cruz-Fiocruz, Instituto Leônidas \& Maria Deane, Manaus, AM, Brasil \\ ${ }^{4}$ Fundação de Medicina Tropical Dr Heitor Vieira Dourado, Gerência de Malária, Manaus, AM, Brasil
}

BACKGROUND Plasmodium vivax, the major cause of malaria in Latin America, has a large subtelomeric multigene family called vir. In the $P$. vivax genome, about $20 \%$ of its sequences are vir genes. Vir antigens are grouped in subfamilies according to their sequence similarities and have been shown to have distinct roles and subcellular locations. However, little is known about vir subfamilies, especially when comes to their functions.

OBJECTIVE To evaluate the diversity, antigenicity, and adhesiveness of Plasmodium vivax VIR-E.

METHODS Vir-E genes were amplified from six P. vivax isolates from Manaus, North of Brazil. The presence of naturally acquired antibodies to recombinant PvBrVIR-E and PvAMA-1 was evaluated by ELISA. Binding capacity of recombinant PvBrVIR-E was assessed by adhesion assay to CHO-ICAM1 cells.

FINDINGS Despite vir-E sequence diversity, among those identified sequences, a representative one was chosen to be expressed as recombinant protein. The presence of IgM or IgG antibodies to PvBrVIR-E was detected in $23.75 \%$ of the study population while the presence of IgG antibodies to PvAMA-1 antigen was $66.25 \%$ in the same population. PvBrVIR-E was adhesive to CHO-ICAM1.

MAIN CONCLUSIONS PvBrVIR-E was antigenic and adhesive to CHO-ICAM1.

Key words: Plasmodium vivax - multigene family - antigenicity - adhesiveness

Malaria is the most disseminated human disease in the world, affecting more than 200 million people every year. ${ }^{(1)}$ Even though malaria rates have been reducing, Plasmodium falciparum remains the largest target of studies, while other Plasmodium species are still neglected. ${ }^{(2)}$ Capable to reach beyond Africa and also present in countries in Asia, Oceania and Americas, $P$. vivax predominates in regions with high population density. ${ }^{(3)}$ This parasite is responsible for more than $70 \%$ of the cases in the Americas and stands as the major cause of malaria in Brazil. ${ }^{(1)}$

Unlike the benign reputation of this parasite, $P$. vivax has the capacity to cause severe diseases. ${ }^{(4,5)}$ While in falciparum malaria the pathogenesis is associated to the parasite ability to adhere to endothelial cells and to non-infected erythrocytes (forming rosettes) ${ }^{(6)}$ in vivax malaria little is known about parasite cytoadherence. ${ }^{(7,8)}$ Variant surface antigens like Plasmodium falciparum

doi: 10.1590/0074-02760210227

Financial support: CNPq (Grant 431403/2016-3), FAPESP (Grant 2017/18611-7).

+ Corresponding author: letusa.albrecht@fiocruz.br

(D) 0000-0001-6406-2057

Received 29 June 2021

Accepted 05 November 2021 erythrocyte membrane protein 1 (PfEMP1) and RIFIN codified by var and rif genes respectively, are responsible for the P. falciparum cytoadherence and linked to parasite ability of escaping from the immune system. ${ }^{(9,10)}$ While these proteins are absent in $P$. vivax another multigene family has been implicated in adhesion and antigenic variation. The $P$. vivax variant genes (vir) family is the largest multifamily of $P$. vivax. Initially, 346 vir genes were identified in $P$. vivax genome, with most of them located at subtelomeric regions and grouped in 12 subfamilies. ${ }^{(11)}$ Later, more than a 1000 vir genes were identified in $P$. vivax genomes. $^{(12)}$ In fact, a previous work from del Portillo and colleagues suggest that $10-20 \%$ of $P$. vivax haploid genome are composed of vir genes. ${ }^{(13)}$ This vast diversity of VIR antigens allows the parasite to evade from the host immune system. ${ }^{(14)}$

VIR proteins can have distinct cellular locations which could imply in different functions. ${ }^{(15)}$ As part of the large vir family, the subfamily E presents a conserved $\mathrm{N}$-terminal domain and polymorphic regions in the C-terminal. ${ }^{(16)}$ Displaying high diversity and being one of the most polymorphic subfamilies, Pexel-like motif are found in $100 \%$ of these sequences, suggesting that these proteins are expressed at the surface of infected reticulocytes. ${ }^{(16)}$ Interested in the great potential of vir genes as a central whole of $P$. vivax pathogenesis, the present study evaluated the genetic diversity, antigenicity and adhesiveness of a VIR-E protein. 


\section{MATERIALS AND METHODS}

Blood sample collection - Blood samples were collected from patients with malaria who were seeking medical care from 2012 to 2014 at Fundação de Medicina Tropical Dr Heitor Vieira Dourado, Manaus, Amazonas State, a low transmission region located in northern Brazil. Blood samples were collected using BD Vacutainer with sodium citrate anticoagulant. The blood was analysed just after its collection using a Sysmex KX21N (Sysmex Corporation-Roche, Japan). A thin blood smear was prepared from each blood sample to determine species of malaria parasites. The samples were confirmed $P$. vivax monospecies infection by Nested-PCR. ${ }^{(17)}$

Amplification and sequencing of vir-e genes - Genomic DNA from whole blood of six patients with vivax malaria was extracted using the phenol:chloroform method. (18) Vir genes from subfamily E (vir-E) were amplified by polymerase chain reaction (PCR) using degenerate virE primers (forward 5'- AA(Y)CAAGAA(W)TTTAT(S) AACTTTGT-3' and reverse 5'- TACC(Y)TATATA(W) CGTTATTAGAGG-3') as previously described by Fernandes-Becerra et al., ${ }^{(14)}$ using Platinum Taq DNA Polymerase High Fidelity (Thermo Fisher Scientific). PCR products were size separated by electrophoresis on a $1 \%$ agarose gel stained with ethidium bromide and visualised under a UV light and purified using Wizard $^{\circledR} S V$ Gel and PCR Clean-Up System (Promega). Each amplified sequence was inserted into pGEM-T Easy Vector (Promega). Sequencing reactions were performed with T7 and SP6 primers and plasmids were sequenced using ABI 3100 (Applied Biosystems, CA, USA).

Sequence analysis - The quality of all acquired sequences was checked by Phred quality above 20 in a sliding window of $300 \mathrm{bp} .^{(19)}$ Plasmid sequences were trimmed out and the sequences were assembled using pregap4, present in the Staden Package. ${ }^{(20)}$ All Brazilian vir-e sequences identified were submitted to GenBank (GenBank accession numbers:MZ357628-MZ357688).

Nucleotide and amino acid sequences were aligned and analysed using ClustalX. ${ }^{(21)}$ A dendrogram was created by ClustalX based on VIR-E amino acid sequences. Antigenicity of VIR-E sequences were analysed using VaxiJen predictor at threshold 0.5. ${ }^{(22)}$

Cloning, recombinant expression and purification of $P$. vivax antigens - One vir-E sequence was chosen to be recombinant expressed in bacterial system and from now on is denominated PvBrVIR-E. The sequence was cloned into pGEX-4T-3 and transformed into competent Escherichia coli STAR BL21 (DE3). The expression was inducted with $0.5 \mathrm{mM}$ isopropyl- $\beta$-D-1thiogalactopyranoside (IPTG) at $18^{\circ} \mathrm{C}$, overnight. Bacterial cultures were then centrifuged at $6.000 \mathrm{~g}$ for $10 \mathrm{~min}$ at $4^{\circ} \mathrm{C}$ and the pellets were lysed using lysis buffer ( 10 $\mathrm{mM}$ Tris-HCl pH 8, $150 \mathrm{mM} \mathrm{NaCl}, 1 \mathrm{mM}$ EDTA) with protease inhibitor (cOmplete ${ }^{\mathrm{TM}}$, Mini, EDTA-free Protease Inhibitor Cocktail Roche) and incubated at $4^{\circ} \mathrm{C}$ for 1 $\mathrm{h}$. Bacteria culture were than disrupted using an M-110L Pneumatic High Shear Fluids Processor (Microfluides).
The soluble fraction was purified using Glutathione Sepharose $4 B$ (GE Healthcare) following the manufacturer's instructions. Protein expression was analysed by sodium dodecyl sulphate-polyacrylamide gel electrophoresis (SDS-PAGE) and western blot. Recombinant $P$. vivax apical membrane antigen 1 variant 5 (PvAMA1V5) and PvAMA1V16 were expressed and purified according as previously described. ${ }^{(23)}$

Detection of naturally acquired antibodies to P. vivax antigens - The presence of immunoglobulin G (IgG) against PvBrVIR-E, PvAMA1V5 and PvAMA1V16 and IgM to PvBrVIR-E recombinant proteins were measured in 80 plasma samples by in-house indirect enzyme-linked immunosorbent assay (ELISA) as previously described. (23) The optical density (OD) was measured at $490 \mathrm{~nm}$ with wavelength correction at $595 \mathrm{~nm}$ using microplate reader (BioTek, Winooski, Vermont, USA). All plates had controls with anti-GST primary antibody (anti-GST control). Twenty plasma samples from healthy individuals from a nonendemic region were used as negative control. The absorbance values were normalised using OD values from anti-GST. The OD cut-off values were 1.0 for PvBrVIR-E IgM, 1.12 for PvBrVIR-E IgG, 0.8 for PvAMA1V5 and 0.85 for PvAMA1V16. Finally, the reactivity index (RI) was obtained from the ratio of absorbance value of each sample and the cut-off value (mean plus three standard deviation of the negative control absorbances). RIs $>1$ were considered positive. Since the proteins were expressed with a GST tag, to avoid bias produced by a possible reactivity of the GST tag, absorbance values of recombinant GST against each plasma sample were evaluated and subtracted from absorbance values of each sample. The OD values to GST ranged from 0 to 0.4 to $\operatorname{IgM}$ and 0 to 0.55 to $\operatorname{IgG}$ antibodies.

Binding assay of PvBrVIR-E to CHO-ICAMI - Chinese hamster ovary $(\mathrm{CHO})$ cells expressing constitutively ICAM1 were grown in RPMI 1640 medium (Sigma) and supplemented with $10 \%$ foetal bovine serum (FBS) and gentamicin, at $37^{\circ} \mathrm{C}$, in an atmosphere of $5 \% \mathrm{CO} 2$. Cells were blocked with blocking solution [phosphatebuffered saline (PBS) solution containing 2\% FBS] for $30 \mathrm{~min}$, at $37^{\circ} \mathrm{C}$. Following the blockade, cells were incubated for $1 \mathrm{~h}$ at $37^{\circ} \mathrm{C}$ with $10 \mu \mathrm{g}$ of recombinant PvBrVIR-E or GST proteins. Subsequently, cells were washed three times with blocking solution and then the polyclonal $\alpha$-GST (Sigma-Aldrich) produced in rabbit (dilution 1:200) was added to the wells. Cells were again washed three times with blocking solution and incubated with 1:100 anti-rabbit antibody conjugated to Alexa488 (Thermo Fisher Scientific). Next, cells were evaluated on Leica DMI6000 fluorescence microscopy.

Statistical analyses - To assess whether there were paired differences between antibody response to the recombinant proteins, Friedman test was applied, followed by pairwise comparisons using Nemenyi post-hoc test. Spearman's correlation coefficient (rho) was estimated and the Spearman's test was applied to inspect the relationship between PvBrVIR-E, PvAMA1V5 and PvAMA1V16 reactivity indexes and the following hae- 
matological parameters: platelets levels, total lymphocytes, neutrophils number of red blood cells, hematocrit and hemoglobin. Additionally, based on haematological parameters, two qualitative variables were derived: anaemia and thrombocytopenia, considered present, respectively, if hemoglobin levels was under $12 \mathrm{~g} / \mathrm{dL}$ and if platelet count was lower than $150 \times 10^{3}$ per $\mu \mathrm{L}$. The reactivity index of each recombinant protein was compared between the categories (present or absent) of these variables through two sample Wilcoxon test. All tests were applied considering a 5\% significance level.

Ethics - Ethical permission for the study was obtained by the local ethical committee in Manaus, Amazonas State, Brazil (CAAE-0044.0.114.000-11). Samples were collected after obtaining written consent from all individuals. After blood sampling, patients were treated for malaria according to national guidelines.

\section{RESULTS}

Genetic diversity of $P$. vivax vir-E - P. vivax vir-E sequences were amplified from six Brazilian isolates. PCR fragments were cloned into a plasmid vector and sequenced. After quality control analyses and sequence assembly, a total of 61 vir-E sequences were obtained (median $=11$, range: $2-15$ per isolate), with a size varying from 795 to 864 base pairs each [Supplementary data (Table I)]. The overall nucleotide identity between sequences was $82.55 \%( \pm 14.01)$. Out of these 61 sequences, 19 had a stop codon and were considered as pseudogenes. Among the remained ones, 38 were unique sequences. The overall amino acid identity between sequences was $79.73 \%( \pm 18.93)$. The antigenicity prediction was evaluated for the 38 amino acid sequences and 36 out of 38 were predicted to be antigenic (Vaxijen score $>0.5$ ). Among the antigenic sequences, 7 had scores higher than 0.6 and were considered highly antigenic [Supplementary data (Table II)].

A phylogenetic tree was build based on amino acid sequences of all vir-E translated sequences [Supplementary data (Fig. 1)]. Thirty sequences shared more than $92 \%$ of amino acid identity (mean $97.15 \% \pm 2.04$ ). One of those antigenic sequence (Vaxijen score: 0.6172 ) here for denominated as PvBrVIR-E (GenBank accession number: MZ357651), was chosen as a representative sequence from VIR subfamily $\mathrm{E}$ and it was expressed and evaluated for antigenicity profile.

Study subjects and haematological parameters - A total of 80 infected individuals from Manaus were analysed. The haematological characteristics of the subjects are shown in Table. In this study, 29 (36.25\%) individuals had hemoglobin levels below $12 \mathrm{~g} / \mathrm{dL}$ and were considered anaemic, while $64(80 \%)$ individuals were thrombocytopenic (platelet count lower than $150 \times 10^{3}$ per $\mu \mathrm{L}$ ).

Naturally acquired antibody to PvBrVIR-E - To verify the antigenicity of PvBrVIR-E protein, the presence of naturally acquired $\operatorname{IgG}$ and IgM antibodies against this protein was evaluated. The purified recombinant PvBrVIR-E has a GST tag and an expected size of approximately $58 \mathrm{kDa}$ (32kDa from rPvBrVIR-E itself and $26 \mathrm{kDa}$ from GST tag). The quality and purity of the recombinant protein was checked by western blotting [Supplementary data (Fig. 2)].

Naturally acquired IgM and $\operatorname{IgG}$ antibodies were evaluated in 80 patients infected with $P$. vivax from Manaus (Table). The majority of individuals had no detectable IgM or IgG antibodies to PvBrVIR-E ( $\mathrm{n}=61$, $76.25 \%$ ), while 19 individuals (23.75\%) had IgM or IgG antibodies to PvBrVIR-E. The presence of IgM antibodies against PvBrVIR-E was detected in $12.5 \%(10 / 80)$ of $P$. vivax patients (Fig. 1A). The same frequency $(12.5 \%$, $\mathrm{n}=10$ ) was observed for the presence of anti-PvBrVIRE IgG antibodies (Fig. 1A, Friedman test, followed by pairwise comparisons using Nemenyi post-hoc test, $\mathrm{p}=$ 0.98 ). Only one individual had IgM and IgG antibodies to PvBrVIR-E. The reactivity index values varied from 0 to 3.36 for $\operatorname{IgM}$ and 0 to 3.65 for $\operatorname{IgG}$.

To evaluate if this low frequency of antibodies were specific against PvBrVIR-E antigen, the presence of IgG antibodies against two previously characterised PvAMA1 variants ${ }^{(23)}$ was also investigated. IgG antibodies were detected in 32 out of $80(40 \%)$ individuals for PvAMA1V16 and 50 out of 80 (62.5\%) individuals for PvAMA1V5. Twenty-seven individuals (33.75\%) had no detectable IgG antibodies to any of those PvAMA1 variants (Fig. 1B).

Paired differences in reactivity index were observed between PvAMAl variants, where the variant PvAMA1V5 was more frequently recognised than PvAMA1V16 (Fig. 1B, Nemenyi post-hoc test, p-value $<0.001)$. In a similar way, differences were observed between PvBrVIR-E IgM response and both PvAMA1 variants IgG response (Fig. 1C-D), and PvBrVIR-E IgG response and PvAMA1V5 IgG response (Fig. 1E). However, no difference was observed between PvBrVIR-E IgG and PvAMA1V16 IgG (Fig. 1F, Nemenyi post-hoc test, $\mathrm{p}$-value $=0.079$ ).

TABLE

Haematological data of Plasmodium vivax infected patients

\begin{tabular}{lc}
\hline Parameters & $\begin{array}{c}P . \text { vivax patients } \\
\mathrm{n}=80\end{array}$ \\
\hline Hematocrit $(\%)$, median (IQR) & $40.75,(36.2-43.3)$ \\
\hline Hemoglobin $(\mathrm{g} / \mathrm{dL})$, median $(\mathrm{IQR})$ & $12.75,(11.3-13.8)$ \\
\hline Anaemia, $\mathrm{n}(\%)$ & $29,(36.25)$ \\
\hline RBC $\left(10^{6} / \mu \mathrm{L}\right)$, median (IQR) & $4.57,(4.05-5.0)$ \\
\hline Platelets $\left(10^{3} / \mu \mathrm{l}\right)$, median $(\mathrm{IQR})$ & $89.5,(53.25-137.5)$ \\
\hline Thrombocytopenia, $\mathrm{n}(\%)$ & $64,(80)$ \\
\hline Neutrophils $(\%)$, median $(\mathrm{IQR})$ & $3.4,(2.3-4.5)$ \\
\hline Lymphocytes $\left(10^{3} / \mu \mathrm{l}\right)$, median $(\mathrm{IQR})$ & $0.9,(0.6-1.4)$ \\
\hline
\end{tabular}

Anaemia was considered when hemoglobin levels were under $12 \mathrm{~g} / \mathrm{dL}$. Thrombocytopenia was considered if platelet count was lower than $150 \times 10^{3}$ per $\mu \mathrm{L}$. 
A

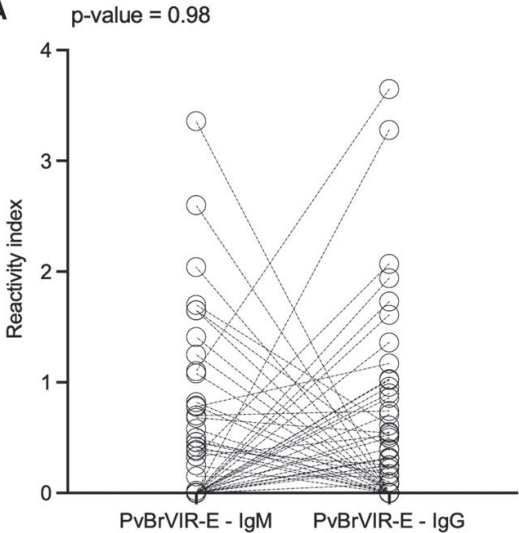

C

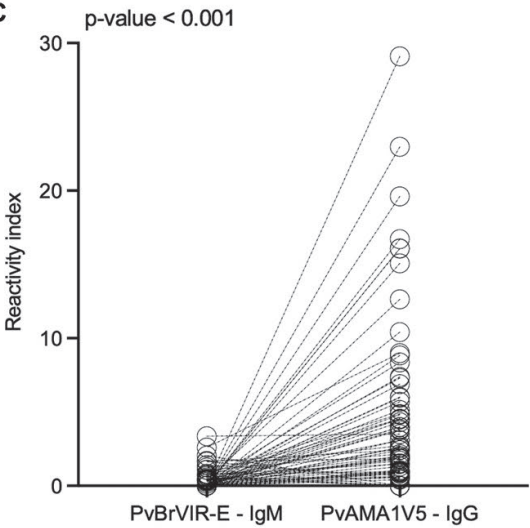

E

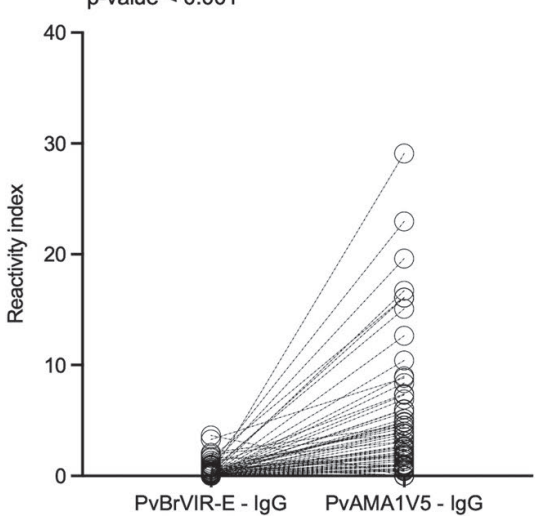

B
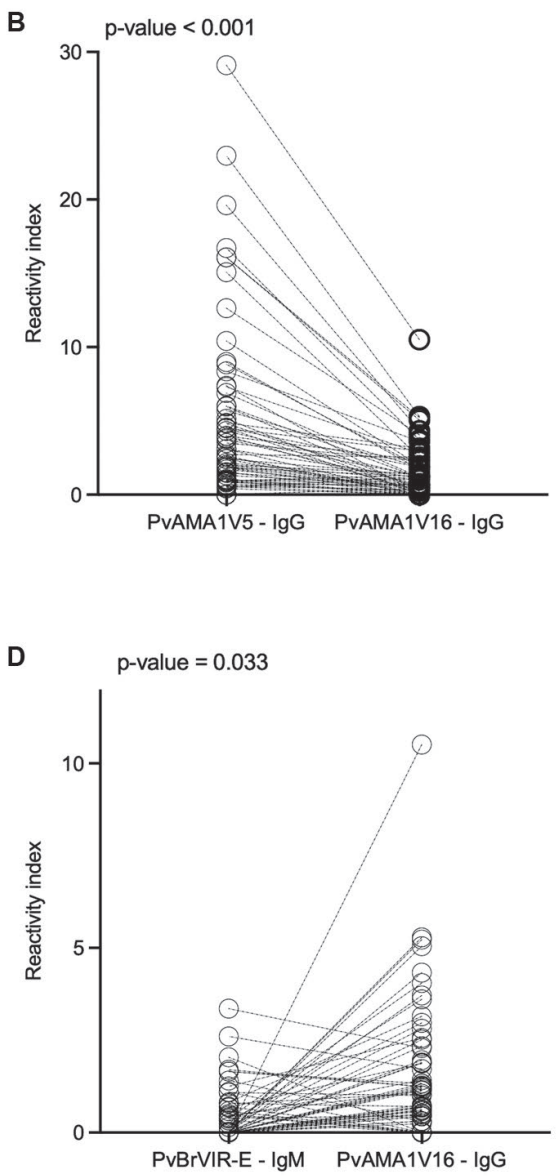

$\mathbf{F}$

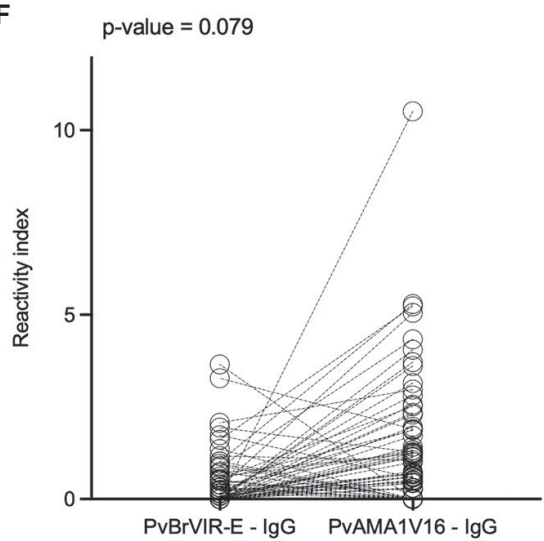

Fig. 1: naturally acquired antibodies against Plasmodium vivax antigens. Human $\operatorname{IgG}$ and $\operatorname{IgM}$ antibody responses to PvBrVIR-E and IgG to PvAMA1 variants were detected in individuals infected with $P$. vivax $(\mathrm{n}=80)$ and pairwise compared: reactivity index to PvBrVIR-E $(A)$. Reactivity index to PvAMA1 variants (B). Reactivity index to PvBrVIR-E IgM compared to PvAMA1V5 (C) and PvAMA1V16 (D). Reactivity index to PvBrVIR-E IgG compared to PvAMA1V5 (E) and PvAMA1V16 (F). Significant differences were calculated by Friedman test, followed by pairwise comparisons using Nemenyi post-hoc test. p-values are indicating in the figures.

The presence of naturally acquired antibodies to PvBrVIR-E and PvAMA1 antigens were evaluated in anae$\mathrm{mic} /$ non-anaemic and thrombocytopenic/non-thrombocytopenic individuals. However, no differences were observed between those groups (Fig. 2A-B), as well as no correlation between haematological parameters and antibody response were observed (Fig. 2C).
PvBrVIR-E binds to endothelial receptor ICAM-1 - Since VIR proteins are implicating to mediate the cytoadhere of $P$. vivax to the endothelial cells, the capacity of the recombinant PvBrVIR-E protein to adhere to CHO cells expressing ICAM1 was evaluated. The recombinant PvBrVIR-E was adherent to CHO-ICAM1 while it was not observed for the recombinant GST protein (Fig. 3). 
A

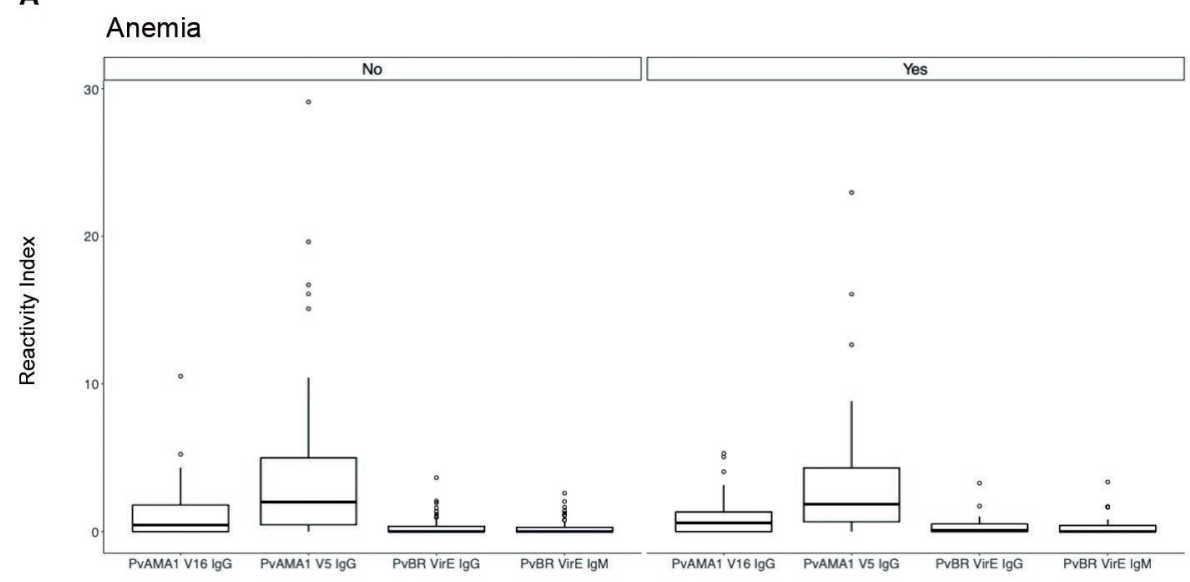

B

Trombocytopenia

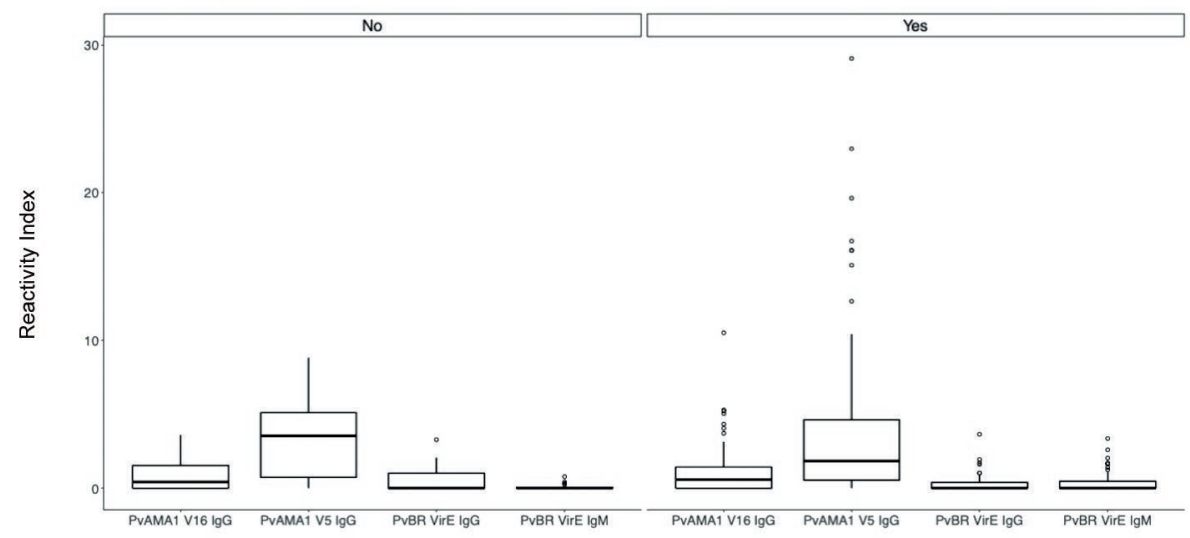

C

\begin{tabular}{|c|c|c|c|c|c|c|c|c|}
\hline & $\begin{array}{l}0 \\
\substack{1 \\
\frac{1}{4}} \\
\frac{1}{2} \\
\frac{1}{2}\end{array}$ & $\frac{\sum_{0}^{\infty}}{\sum_{0}^{1}}$ & 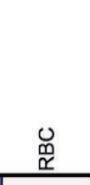 & $\begin{array}{l}\overline{\mathbf{z}} \\
\overline{0} \\
0 \\
00 \\
\underline{0} \\
\end{array}$ & $\begin{array}{l}\stackrel{\circ}{5} \\
\stackrel{5}{1}\end{array}$ & 吉 & 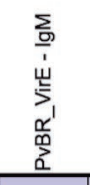 & 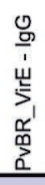 \\
\hline PvAMA1-V16 & 1.00 & 0.74 & -0.03 & -0.02 & -0.08 & -0.05 & 0.17 & 0.14 \\
\hline PvAMA1-V5 & 0.74 & 1.00 & 0.01 & 0.04 & 0.06 & 0.03 & -0.07 & 0.21 \\
\hline RBC & -0.03 & 0.01 & 1.00 & 0.86 & 0.91 & 0.17 & $2.25 \times 10^{-3}$ & -0.12 \\
\hline $\mathrm{HGB} g / \mathrm{dl}$ & -0.02 & 0.04 & 0.86 & 1.00 & 0.94 & 0.13 & 0.02 & -0.16 \\
\hline НСT \% & -0.08 & 0.06 & 0.91 & 0.94 & 1.00 & 0.18 & -0.08 & -0.14 \\
\hline PLT & -0.05 & 0.03 & 0.17 & 0.13 & 0.18 & 1.00 & -0.13 & 0.06 \\
\hline PvBR_VirE - IgM & 0.17 & -0.07 & $2.25 \times 10^{-3}$ & 0.02 & -0.08 & -0.13 & 1.00 & 0.06 \\
\hline PvBR_VirE - lgG & 0.14 & 0.21 & -0.12 & -0.16 & -0.14 & 0.06 & 0.06 & 1.00 \\
\hline
\end{tabular}

Fig. 2: antibody response and their relationship with haematological parameters. Naturally acquired antibody response to PvBrVIR-E and PvAMAlvariants and haematological parameters were evaluated in individuals infected with Plasmodium vivax $(\mathrm{n}=80)$. (A) PvBrVIR-E and PvAMA1 reactivity index distribution in anaemic/ non-anaemic individuals. (B) PvBrVIR-E and PvAMA1 reactivity index distribution in individuals with/without thrombocytopenia. (C) Spearman's correlation coefficient (rho) between antibody response and haematological parameters. 

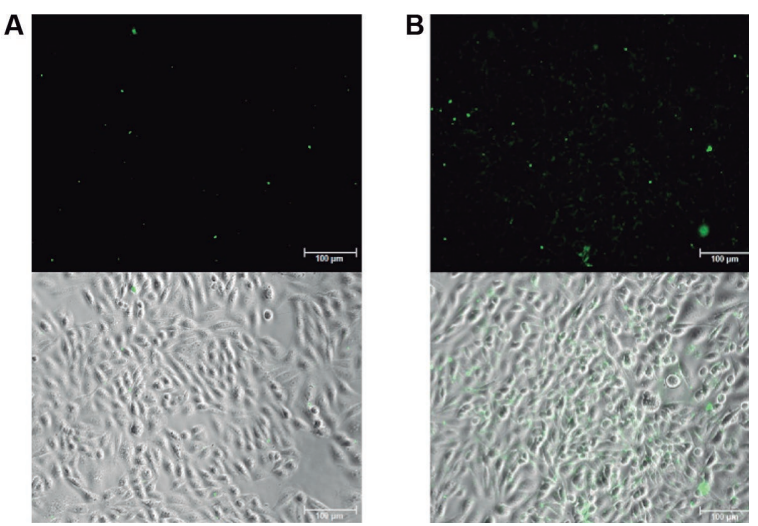

Fig. 3: binding of PvBrVIR-E on CHO-ICAM1. Recombinant GST (A) or recombinant PvBrVIR-E (B) were incubated with CHO-ICAM1 cells and binding was detected by anti-GST on Leica DMI6000 fluorescence microscopy. Green spots represent the protein binding to CHO-ICAM1 cells detected by the fluorescent antibody.

\section{DISCUSSION}

The major virulence antigens in $P$. vivax are the VIR proteins which are codified by genes from the variant vir superfamily. This family is extremely diverse and clustered in subgroups, suggesting distinct localisation and functions. ${ }^{(13,14,24)}$ Among all multigenic families described in Plasmodium species, vir genes are one of the most polymorphic. (25) Among the vir subfamilies the vir-E group presents a PEXEL motif, suggesting that these proteins would reach the surface of the infected reticulocyte. ${ }^{(16)}$ Here, the genetic diversity of this subfamily, as well as its antigenicity and binding capacity were evaluated. When diversity of vir-E gene was assessed, 38 coding genes were identified in isolates. Despite the high variability of vir genes, a phylogenetic analysis showed a cluster of similar sequences whereas most of isolates here analysed had a representative gene, showing some degree of conservation among $P$. vivax Brazilian isolates. Even though almost $20 \%$ of the $P$. vivax genomes codifies VIR antigens, very little was explored about its role. The vir sequences here identified were majority coding sequences and predicted to be antigenic. However, a high percentage of truncate sequences were also found and considered pseudogenes. A high number of pseudogenes are frequently observed in multigenic gene families from $P$. vivax and $P$. falciparum field isolates. ${ }^{(16,26)}$

The host immune response against VIR antigens is not well characterised mainly due to its high polymorphism rates that difficult the amplification of coding sequences and expression of the recombinant proteins for immunoassay. ${ }^{(16)}$ Besides, the lack of a long-term in vitro culture for $P$. vivax is also an obstacle to the comprehension of immunological mechanisms. ${ }^{(27,28)}$ The expressive number of distinct vir genes on annotated genomes is also a challenge in the study of these antigens. ${ }^{(12)}$ Here, a vir-E gene identified from $P$. vivax field isolates from Brazil was recombinant expressed.

As previously observed, VIR proteins are antigenic, but the frequency of individuals that present antibodies against these antigens are quite heterogeneous, de- pending on the VIR antigen and the geographic region evaluated..$^{(29,30)}$ Thus far, the frequency of individuals that had antibodies to recombinant VIR proteins are lower in Brazil when compared to Papua New Guine and Guatemala. ${ }^{(29)}$ Here, $12.5 \%$ of the study population had $\mathrm{IgG}$ antibodies to PvBrVIR-E while in a previous study with populations from Brazil identified antibodies in $26 \%$ and another one found between 1 to $42 \%$ of the population, depending on VIR antigen analysed. When comes specifically to the response to a VIR-E antigen the number of responders drops to $2.5-10 \%{ }^{(30)}$ The frequency of individuals that had $\operatorname{IgM}$ antibodies to PvBrVIR-E was similar for IgG (12.5\%) and only one individual had the presence of both classes of antibodies. It is important to note that all individuals were living in malaria endemic area and therefore exposed to $P$. vivax infection. So far, there is no information about how is the dynamic of the antibody response to VIR antigens and how long they can last.

The low frequency of responders to VIR antigens is quite expected since the diversity of VIR antigens is high and it is still not known which of these antigens are going to be expressed by the parasite. Lesser is known about how long these antibodies could persistent or cross react between different VIR antigens.

Individuals exposed to $P$. vivax frequently have higher response to polymorphic merozoite antigens than to VIR antigens. ${ }^{(30,31)}$ In this study, despite the low frequency of VIR-E responders, most of individuals had antibodies to the microneme antigen PvAMA1 (66.25\%). Due the sample limitation, only IgG antibodies to PvAMA1 variants were evaluated. Similar results were also observed when compared the response to VIR-E antigen and PvAMA1 in different regions from North of Brazil. ${ }^{(30)}$ This difference is most likely due high frequency of polymorphisms found at VIR antigens compared to other polymorphic antigens. ${ }^{(25)}$

Recently, we showed that PvAMA1V5 was more frequently recognised than PvAMA1V16 in populations from distinct locations from North of Brazil. (23) Here, similar results were observed (Fig. 1B), confirming that PvAMA1V5 is more frequently recognised in North of Brazil. The sequence coding PvAMA1V5 was also more frequently identified in a population from Manaus and several polymorphic sites between PvAMA1V5 and PvAMA1V16 were described. ${ }^{(23)}$

In fact, polymorphisms can have a direct impact on the protein structure and therefore, on the B cell epitope. So far, the crystal structure of VIR antigens has not been solved and since these antigens are very polymorphic is difficult to model them based to any other known antigens. Although there are more tools to predict antigenicity, it is relevant to point out that Vaxijen2.0, different from other tools to predict antigenicity, used not only bacteria and virus sequences to develop it, but also parasites proteins yields trustworthy results. ${ }^{(22)}$ In contrast, Vaxign2, Vaxign-ML and Vaxijen3.0 although developed as a machine learning (ML) model; which has better results; their training sets were exclusively with bacterial proteins. ${ }^{(32,33,34)}$ Therefore, they are not to be trusted in regards of antigenicity of a protozoa parasite. 
Dalsass and colleagues noted that a satisfactory way of predicting antigenicity would be to combine the results of a filtering method, such as Vaxijen2.0, with another using ML. ${ }^{(35)}$ Unfortunately, there are no ML based predictors trained with protozoa data, so our most truthfully results are those of Vaxijen2.0.

Few studies have been explored the function of VIR proteins. In part, this lack of data is explained by the difficulties while working with $P$. vivax. As recently observed in several studies, $P$. vivax can adhere to endothelial receptors and form rosettes in a similar fashion as P. falciparum. ${ }^{(36,37)}$ However, unlike P. falciparum, PfEMP1 and RIFIN antigens are absent in $P$. vivax. Therefore, some other antigens must be implicated in the adhesion phenomena. In fact, a VIR antigen from subfamily $\mathrm{C}$ have been demonstrated to adhere to ICAM$1{ }^{(15)}$ Here, the adhesion to the PvBrVIR-E antigen to CHO-ICAM1 was also observed, suggesting that this subfamily, as for subfamily C, can be implicated in adhesion phenomenon. Nevertheless, extracellular vesicles derived from plasma of $P$. vivax infected patient were taken up by human spleen fibroblast and upregulated the expression of ICAM-1. ${ }^{(38)}$

Taking all together, here we showed that vir-E subfamily codifies antigenic VIR-E protein and it is recognised by a small part of $P$. vivax infected individuals. Nonetheless, the recombinant PvBrVIR-E was also adherent to CHO-ICAM1 cells. These data open avenues for the study of variant antigens in $P$. vivax and their implications on immunity and adhesion.

\section{ACKNOWLEDGEMENTS}

To the people who agreed to participate in this study, to the field team at Fundação de Medicina Tropical Dr Heitor Vieira Dourado (FMT-HVD) in Manaus. The authors also thank the Program for Technological Development in Tools for Health-PDTIS FIOCRUZ at the Carlos Chagas Institute, Fiocruz-Paraná.

\section{AUTHORS' CONTRIBUTION}

APS, ABI, NCB, LB and SF performed the experiments; NCB, HGS, JHG, SCPL, MVGL, FTMC and LA participated in the data analyses; LA conceived the study; NCB, LB, HGS and LA wrote the final version of the manuscript. All authors read and approved the final manuscript. The authors declare that they have no competing interests.

\section{REFERENCES}

1. WHO - World Health Organization. World Malaria Report. 2020. Available from: https://www.who.int/publications/i/ item/9789240015791.

2. Gething PW, Elyazar IR, Moyes CL, Smith DL, Battle KE, Guerra $\mathrm{CA}$, et al. A long neglected world malaria map: Plasmodium vivax endemicity in 2010. PLoS Negl Trop Dis. 2012; 6(9):e1814. doi:10.1371/journal.pntd.0001814.

3. Howes RE, Battle KE, Mendis KN, Smith DL, Cibulskis RE, Baird JK, et al. Global epidemiology of Plasmodium vivax. Am J Trop Med Hyg. 2016; 95(Suppl. 6): 15-34. doi:10.4269/ajtmh.16-0141.

4. Kojom Foko LP, Arya A, Sharma A, Singh V. Epidemiology and clinical outcomes of severe Plasmodium vivax malaria in India. $\mathrm{J}$ Infect. 2021; 82(6): 231-46. doi:10.1016/j.jinf.2021.03.028.
5. Lacerda MV, Mourão MP, Alexandre MA, Siqueira AM, Maga1hães BM, Martinez-Espinosa FE, et al. Understanding the clinical spectrum of complicated Plasmodium vivax malaria: a systematic review on the contributions of the Brazilian literature. Malar J. 2012; 11: 12. doi:10.1186/1475-2875-11-12.

6. Wahlgren M, Goel S, Akhouri RR. Variant surface antigens of Plasmodium falciparum and their roles in severe malaria. Nat Rev Microbiol. 2017; 15(8): 479-91. doi:10.1038/nrmicro.2017.47.

7. Bittencourt N, Bertolla LP, Albrecht L. Insights on rosetting phenomenon in Plasmodium vivax malaria. Curr Clin Microbiol Rep. 2021; 8: 1-7. doi:https://doi.org/10.1007/s40588-020-00155-3.

8. Totino PR, Lopes SC. Insights into the cytoadherence phenomenon of. Front Immunol. 2017; 8: 1148. doi:10.3389/fimmu.2017.01148.

9. Jensen AR, Adams Y, Hviid L. Cerebral Plasmodium falciparum malaria: the role of PfEMP1 in its pathogenesis and immunity, and PfEMP1-based vaccines to prevent it. Immunol Rev. 2020; 293(1): 230-52. doi:10.1111/imr.12807.

10. Quintana MDP, Ch'ng JH, Moll K, Zandian A, Nilsson P, Idris ZM, et al. Antibodies in children with malaria to PfEMP1, RIFIN and SURFIN expressed at the Plasmodium falciparum parasitized red blood cell surface. Sci Rep. 2018; 8(1): 3262. doi:10.1038/ s41598-018-21026-4.

11. Carlton JM, Adams JH, Silva JC, Bidwell SL, Lorenzi H, Caler $\mathrm{E}$, et al. Comparative genomics of the neglected human malaria parasite Plasmodium vivax. Nature. 2008; 455(7214): 757-63. doi:10.1038/nature07327.

12. Auburn S, Böhme U, Steinbiss S, Trimarsanto H, Hostetler J, Sanders MJ, et al. A new Plasmodium vivax reference sequence with improved assembly of the subtelomeres reveals an abundance of pir genes. Wellcome Open Res. 2016; 1: 4. doi:10.12688/wellcomeopenres.9876.1.

13. del Portillo HA, Fernandez-Becerra C, Bowman S, Oliver K, Preuss M, Sanchez CP, et al. A superfamily of variant genes encoded in the subtelomeric region of Plasmodium vivax. Nature. 2001; 410(6830): 839-42. doi:10.1038/35071118.

14. Fernandez-Becerra C, Pein O, de Oliveira TR, Yamamoto MM, Cassola AC, Rocha C, et al. Variant proteins of Plasmodium vivax are not clonally expressed in natural infections. Mol Microbiol. 2005; 58(3): 648-58. doi:10.1111/j.1365-2958.2005.04850.x.

15. Bernabeu M, Lopez FJ, Ferrer M, Martin-Jaular L, Razaname A, Corradin G, et al. Functional analysis of Plasmodium vivax VIR proteins reveals different subcellular localizations and cytoadherence to the ICAM-1 endothelial receptor. Cell Microbiol. 2012; 14(3): 386-400. doi:10.1111/j.1462-5822.2011.01726.x.

16. Merino EF, Fernandez-Becerra C, Durham AM, Ferreira JE, Tumilasci VF, d'Arc-Neves J, et al. Multi-character population study of the vir subtelomeric multigene superfamily of Plasmodium vivax, a major human malaria parasite. Mol Biochem Parasitol. 2006; 149(1): 10-6. doi:10.1016/j.molbiopara.2006.04.002.

17. Snounou G, Singh B. Nested PCR analysis of Plasmodium parasites. Methods Mol Med. 2002; 72: 189-203. doi:10.1385/1-59259271-6:189.

18. Moll K, Kaneko A, Scherf A, Wahlgren M, editors. Methods in Malaria Research. 6th ed. Gasgow: EviMalarR; 2013.

19. Ewing B, Green P. Base-calling of automated sequencer traces using phred. II. Error probabilities. Genome Res. 1998; 8(3): 186-94.

20. Staden R, Beal KF, Bonfield JK. The Staden package, 1998. Methods Mol Biol. 2000; 132: 115-30.

21. Thompson JD, Gibson TJ, Plewniak F, Jeanmougin F, Higgins DG. The CLUSTAL_X windows interface: flexible strategies for multiple sequence alignment aided by quality analysis tools. Nucleic Acids Res. 1997; 25(24): 4876-82. doi:10.1093/nar/25.24.4876. 
22. Doytchinova IA, Flower DR. VaxiJen: a server for prediction of protective antigens, tumour antigens and subunit vaccines. BMC Bioinformatics. 2007; 8: 4. doi:10.1186/1471-2105-8-4.

23. Bittencourt NC, Silva ABIE, Virgili NS, Schappo AP, Gervásio JH, Pimenta TS, et al. Plasmodium vivax AMA1: implications of distinct haplotypes for immune response. PLoS Negl Trop Dis. 2020; 14(7): e0008471. doi:10.1371/journal.pntd.0008471.

24. Lopez FJ, Bernabeu M, Fernandez-Becerra C, del Portillo HA. A new computational approach redefines the subtelomeric vir superfamily of Plasmodium vivax. BMC Genomics. 2013; 14: 8 . doi:10.1186/1471-2164-14-8.

25. Neafsey DE, Galinsky K, Jiang RH, Young L, Sykes SM, Saif $\mathrm{S}$, et al. The malaria parasite Plasmodium vivax exhibits greater genetic diversity than Plasmodium falciparum. Nat Genet. 2012; 44(9): 1046-50. doi:10.1038/ng.2373.

26. Albrecht L, Merino EF, Hoffmann EH, Ferreira MU, Ferreira RG, Osakabe AL, et al. Extense variant gene family repertoire overlap in Western Amazon Plasmodium falciparum isolates. Mol Biochem Parasitol. 2006; 150(2): 157-65. doi:10.1016/j.molbiopara.2006.07.007.

27. Gunalan K, Rowley EH, Miller LH. A way forward for culturing Plasmodium vivax. Trends Parasitol. 2020; 36(6): 512-9. doi:10.1016/j.pt.2020.04.002.

28. Costa FT, Lopes SC, Albrecht L, Ataíde R, Siqueira AM, Souza RM, et al. On the pathogenesis of Plasmodium vivax malaria: perspectives from the Brazilian field. Int J Parasitol. 2012; 42(12): 1099-105. doi:10.1016/j.ijpara.2012.08.007.

29. Requena P, Rui E, Padilla N, Martínez-Espinosa FE, Castellanos ME, Bôtto-Menezes C, et al. Plasmodium vivax VIR proteins are targets of naturally-acquired antibody and $\mathrm{T}$ cell immune responses to malaria in pregnant women. PLoS Negl Trop Dis. 2016; 10(10): e0005009. doi:10.1371/journal.pntd.0005009.

30. Oliveira TR, Fernandez-Becerra C, Jimenez MC, Del Portillo HA, Soares IS. Evaluation of the acquired immune responses to Plasmodium vivax VIR variant antigens in individuals living in malaria-endemic areas of Brazil. Malar J. 2006; 5: 83. doi:10.1186/1475-2875-5-83.

31. Lee S, Choi YK, Goo YK. Humoral and cellular immune response to Plasmodium vivax VIR recombinant and synthetic antigens in individuals naturally exposed to $P$. vivax in the Republic of Korea. Malar J. 2021; 20(1): 288. doi:10.1186/s12936-021-03810-2.

32. Ong E, Cooke MF, Huffman A, Xiang Z, Wong MU, Wang H, et al. Vaxign2: the second generation of the first Web-based vaccine design program using reverse vaccinology and machine learning. Nucleic Acids Res. 2021; 49(W1): W671-8. doi:10.1093/nar/gkab279.

33. Zaharieva N, Dimitrov I, Flower DR, Doytchinova I. VaxiJen dataset of bacterial immunogens: an update. Curr Comput Aided Drug Des. 2019; 15(5): 398-400. doi:10.2174/1573409915666190 318121838 .

34. Dimitrov I, Zaharieva N, Doytchinova I. Bacterial immunogenicity prediction by machine learning methods. Vaccines (Basel). 2020; 8(4): 709. doi:10.3390/vaccines8040709.

35. Dalsass M, Brozzi A, Medini D, Rappuoli R. Comparison of open-source reverse vaccinology programs for bacterial vaccine antigen discovery. Front Immunol. 2019; 10: 113. doi:10.3389/ fimmu.2019.00113.

36. Carvalho BO, Lopes SC, Nogueira PA, Orlandi PP, Bargieri DY, Blanco TC, et al. On the cytoadhesion of Plasmodium vivax-infected erythrocytes. J Infect Dis. 2010; 202(4): 638-47. doi: $10.1086 / 654815$.

37. Albrecht L, Lopes SC, da Silva AB, Barbosa V, Almeida RP, Siqueira AM, et al. Rosettes integrity protects Plasmodium vivax of being phagocytized. Sci Rep. 2020; 10(1): 16706. doi:10.1038/ s41598-020-73713-w.

38. Toda H, Diaz-Varela M, Segui-Barber J, Roobsoong W, Baro B, Garcia-Silva $\mathrm{S}$, et al. Plasma-derived extracellular vesicles from Plasmodium vivax patients signal spleen fibroblasts via NF-kB facilitating parasite cytoadherence. Nat Commun. 2020; 11(1): 2761. doi:10.1038/s41467-020-16337-y. 